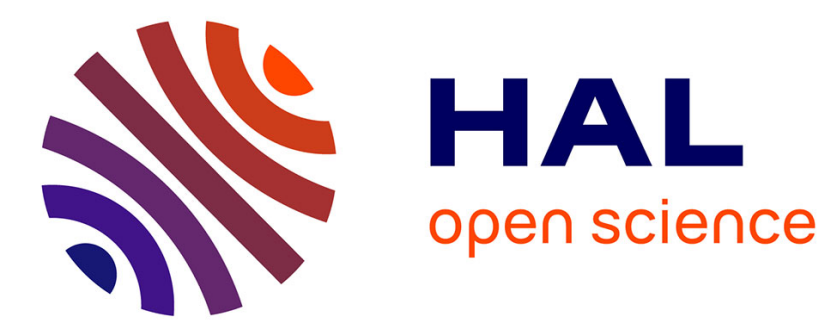

\title{
Connectivity-consistent mapping method for 2-D discrete fracture networks
}

Delphine Roubinet, Jean-Raynald de Dreuzy, Philippe Davy

\section{To cite this version:}

Delphine Roubinet, Jean-Raynald de Dreuzy, Philippe Davy. Connectivity-consistent mapping method for 2-D discrete fracture networks. Water Resources Research, 2010, 46, pp.W07532. 10.1029/2009WR008302 . insu-00604949

\section{HAL Id: insu-00604949 \\ https://hal-insu.archives-ouvertes.fr/insu-00604949}

Submitted on 30 Jun 2011

HAL is a multi-disciplinary open access archive for the deposit and dissemination of scientific research documents, whether they are published or not. The documents may come from teaching and research institutions in France or abroad, or from public or private research centers.
L'archive ouverte pluridisciplinaire $\mathbf{H A L}$, est destinée au dépôt et à la diffusion de documents scientifiques de niveau recherche, publiés ou non, émanant des établissements d'enseignement et de recherche français ou étrangers, des laboratoires publics ou privés. 


\section{Connectivity-consistent mapping method for 2D discrete fracture networks}

2 Delphine Roubinet, Jean-Raynald de Dreuzy and Philippe Davy

3 Geosciences Rennes, UMR CNRS 6118, Université de Rennes I, Rennes, France

\section{Abstract}

5 We present a new flow computation method in 2D Discrete Fracture Networks (DFN) intermediary

6 between the classical DFN flow simulation method and the projection onto continuous grids. The

7 method divides the simulation complexity by solving for flows successively at a local mesh scale and

8 at the global domain scale. At the mesh scale, flows are determined by classical DFN flow

9 simulations and approximated by an Equivalent Hydraulic Matrix (EHM) relating heads and flow

10 rates discretized on the mesh borders. Assembling the Equivalent Hydraulic Matrices provides for a

11 domain-scale discretization of the flow equation. The Equivalent Hydraulic Matrices transfer the

12 connectivity and flow structure complexities from the mesh scale to the domain scale. Compared to

13 existing geometrical mapping or equivalent tensor methods, the EHM method broadens the

14 simulation range of flow to all types of $2 \mathrm{D}$ fracture networks both below and above the

15 Representative Elementary Volume (REV). Additional computation linked to the derivation of the

16 mesh-scale Equivalent Hydraulic Matrices increases the accuracy and reliability of the method.

17 Compared to DFN methods, the EHM method first provides a simpler domain-scale alternative

18 permeability model. Second, it enhances the simulation capacities to larger fracture networks where

19 flow discretization on the DFN structure yields system sizes too large to be solved using the most

20 advanced multigrid and multifrontal methods. We show that the EHM method continuously moves

21 from the DFN method to the tensor representation as a function of the mesh-scale discretization. The

22 balance between accuracy and model simplification can be optimally controlled by adjusting the 23 domain-scale and mesh-scale discretizations. 


\section{Introduction}

26 Fractured media has been classically modeled using either Discrete Fracture Network (DFN) or

27 Stochastic Continuum (SC) approaches [Neuman, 2005]. Both approaches have their own advantages 28 and drawbacks [Hsieh, 1998]. First, they differ by their underlying permeability structure and their

29 capacity of being specified by existing field data [Hsieh, 1998]. The DFN approach easily accounts

30 for extensive fracture characterization [Cvetkovic et al., 2004; Davy et al., 2006] while the SC

31 approach copes more consistently with hydraulic data [Ando et al., 2003]. Second, the simulation of

32 hydraulic processes requires the development of specific methods using the DFN approach whereas 33 only standard discretization schemes are required with the SC approach. Third, because the SC 34 approach simplifies the fracture network structure, it is generally less computationally demanding 35 than the DFN method. Hybrid approaches have been developed to combine the advantages of the 36 DFN and SC approaches. Most of them use a DFN approach at the onset for building equivalent 37 heterogeneous continuous models mapping either the smallest fractures [Lee et al., 2001] or all 38 fractures in the case of the Fracture Continuum Model (FCM) [Botros et al., 2008; Bourbiaux et al., 39 1998; Jackson et al., 2002; Reeves et al., 2008; Svensson, 2001]. Fracture Continuum Models aim at 40 benefiting both from the structure complexity of DFNs and from the simulation and computational 41 simplicities of continuous media. The objective is often to use the FCM approximation as a basis for 42 simulating more computationally demanding transient or multiphase flows [Bourbiaux et al., 1998; 43 Karimi-Fard et al., 2006].

44 The quality of the FCM models critically depends on the derivation of the block-scale permeabilities 45 from the DFNs, i.e. on the mapping of the fracture network onto the continuum grid. The block is 46 considered here as the elementary cell of the continuum grid. Block-scale permeabilities are obtained 47 either from geometrical characteristics [Botros et al., 2008; Svensson, 2001] or through block-scale 
48 numerical simulations of flow [Jackson et al., 2002]. Potential errors stem from differences between

49 the derived scalar or tensor permeabilities and the effective flows within the block. They arise from

50 the difficulty to account for complex fracture connectivity on a broad range of scales. For mapping

51 based on geometrical rules, errors decrease with finer discretization whereas for mapping based on

52 hydraulic computation of the equivalent permeability tensor, errors increase below the

53 Representative Elementary Volume [Long et al., 1982]. Jackson et al. [2002] corrected part of the

54 latter error by using a larger simulation zone, namely the "guard zone", designed to remove dummy

55 additional fracture connectivity with the sides of the block. FCMs keep the general connectivity

56 structure above the scale of the block but remove most of the connectivity effects at lower scales.

57 This results in less flow localization at the block scale and in difficulties in defining an equivalent

58 block permeability tensor. A simple assessment criterion of the relevance of the tensor representation

59 is the difference between flows on opposite block faces. They are equal in the tensor representation.

60 Their difference is expected to increase steeply for complex networks below the REV scale as shown

61 in the example of Figure 1. To avoid handling complex connectivity at the block scale, existing FCM

62 methods are applied either at scales close to the smallest fractures modeled [Botros et al., 2008;

63 Reeves et al., 2008] or at scales larger than the Representative Elementary Volume (REV)

64 [Durlofsky, 1991; Jackson et al., 2002]. The first methods, i.e. the methods applicable to scales close

65 to the smallest fracture modeled, represent permeability by a scalar or a diagonal tensor. They

66 require fine grids for fractured medium representation but can be highly accurate for not too dense

67 fracture networks [Botros et al., 2008]. The second methods, i.e. the methods applicable to scales

68 larger than the REV, represent permeability by an anisotropic full tensor defined by three 2D

69 parameters $K_{x x}, K_{y y}$ and $K_{x y}=K_{y x}$. They require the a priori knowledge of the REV and are hence more

70 suited to dense fracture networks. Their drawbacks are the strong homogenization of flow, their

71 applicability to a restricted scale range and the increase of the numerical error with the refinement of

72 discretization. 
73 None of these methods applies between the scale of the smallest fractures modeled and the REV, a

74 scale range that spans orders of magnitude for multiscale fracture networks (i.e. fracture networks for

75 which the fracture-length distribution is a power law) [Bonnet et al., 2001; de Dreuzy et al., 2001b].

76 In fact, this scale range extends at least from the connectivity scale to the REV scale. The

77 connectivity scale is the scale at which networks are just connected. It ranges from meters to

78 kilometers [Berkowitz et al., 2000; Davy et al., 2009]. Because of the fracture transmissivity

79 variability, the REV scale can be one to three orders of magnitude larger than the connectivity scale

80 [Baghbanan and Jing, 2007; de Dreuzy et al., 2001a; 2002]. Extending at least from the scales

81 contributing to connectivity to the REV scale, the scale range of fractures contributing to flow covers

82 several orders of magnitude from the meter to the kilometer scale. For this scale range, the only

83 available flow simulation method is the DFN method. The DFN flow simulation method, however, is

84 limited in terms of fracture number and domain size. The limiting step arises when solving the linear

85 system issued from the flow discretization on the network structure. With traditional system-solving

86 methods like the conjugate gradient, limitations stemmed from computation time. However, the new

87 numerical methods like the multifrontal or algebraic multigrid method, as implemented in

88 UMFPACK [Davis, 2004] and HYPRE [Falgout et al., 2005], are orders of magnitude faster but

89 require additional memory [de Dreuzy and Erhel, 2002]. Their sole limitation is the computer

90 memory. As a rule of thumb, they can solve at most a linear system of rank one million in a couple

91 of minutes on a personal workstation (Pentium Xeon, $3 \mathrm{GHz}, 8 \mathrm{Go}$ ). Consequently, improving

92 simulation capacities is not about speeding up the method but about enabling simulations otherwise

93 impossible because of memory requirements. We will thus look in this paper at the numerical

94 memory complexity rather than at the numerical time complexity. Our longer-term strategy is to use

95 parallel computing for performing Monte-Carlo simulations while sequential individual simulations

96 remain sequential [Erhel et al., 2009]. This ensures scalability and a minimum of parallel computing

97 implementation. 
98 We propose a new FCM method for the scale range where no existing FCM method is applicable.

99 Like with the previously-cited FCM methods, the objective is to simplify the domain-scale numerical

100 scheme and computations while keeping the complexity of the DFN structure. The new method

101 divides the simulation complexity by solving for flows successively at the local block scale and at

102 the global domain scale. At the block scale, flows are determined by classical DFN flow simulations

103 and approximated by an Equivalent Hydraulic Matrix (EHM) relating heads and flow rates 104 discretized on the mesh borders. Assembling the Equivalent Hydraulic Matrices allows for a domain105 scale discretization of the flow equation. The Equivalent Hydraulic Matrices transfer the connectivity 106 and flow structure complexities from the block scale to the domain scale. The method is similar to 107 Boundary Element Methods [Dershowitz and Fidelibus, 1999] as it relates heads and flow rates on 108 the block borders. As the Equivalent Hydraulic Matrices are determined at the block scale by DFN 109 simulations, we show that the method is systematically applicable regardless of the scale, fracture 110 density and fracture-length and transmissivity distributions. The method accuracy and complexity are 111 given by the level of discretization of the block borders and of the domain. We call this method the 112 Equivalent Hydraulic Matrices (EHM) method as heads and flow rates on the block borders are 113 linearly linked by a matrix representing the block-scale hydraulic properties rather than by a scalar or 114 a tensor permeability. This article describes the EHM method (section 2), shows its results compared 115 to existing methods (section 3) and discusses its performance (section 4).

\section{2. The Equivalent Hydraulic Matrices method}

117 This section defines the EHM method. Once the domain meshed into elementary blocks, the 118 principle of the EHM method is to express the block-scale hydraulic properties by a linear 119 relationship between discretized flow rates and heads on the block borders. This expression will 120 replace the scalar or tensor models used in classical FCM models. With $\boldsymbol{p}_{\boldsymbol{k}}$ as the discretization 121 points (also called poles) of the block numbered $k$, the vector of flow rates $\boldsymbol{\phi}_{\boldsymbol{k}}$ and heads $\boldsymbol{H}_{\boldsymbol{k}}$ on these 
points are related by the following linear relationship:

$$
\phi_{k}=A_{k} \cdot H_{k}
$$

124 The block matrix $\boldsymbol{A}_{\boldsymbol{k}}$ contains sub-block scale connectivity information and can be considered as the 125 block-scale constitutive relationship. It is obtained by performing block-scale flow simulations on 126 the DFN. Once obtained, the block-scale matrices $\boldsymbol{A}_{\boldsymbol{k}}$ are used for simulating flow rates at the system 127 scale by imposing the continuity of heads and flow rates across the block borders. Relationship (1) 128 differs a priori from Darcy's law by its relating flow rates to heads and not to head gradients. This is 129 only a surface difference since the construction method (section 2.2) and the resulting properties of 130 matrices $\boldsymbol{A}_{\boldsymbol{k}}$ (Appendix A) ensure a dependence of the flow rates on head gradients.

\section{$131 \quad$ 2.1. Discretization}

132 Discretization is made up of two parts consisting in discretization of the domain into elementary 133 blocks (classical meshes) and discretization of block borders into poles. The first discretization 134 consists in defining the mesh of the Fracture Continuum Model. We use hereafter a regular grid even 135 though the EHM method can cope with irregular meshes. Each mesh cell will be called a block. The 136 block contains a subset of the fracture network, i.e. a sub-network, the intersections of which with 137 the block limits are denoted $\boldsymbol{m}_{\boldsymbol{k}} \cdot \boldsymbol{m}_{\boldsymbol{k}}(i)$ is the $i^{\text {th }}$ intersection of block $k$. The second discretization 138 consists in splitting up the block borders into segments of constant length $d_{\text {block }}$, the discretization of 139 each border starting at the border corner. Each segment contains either zero, one or more than one

140 fracture border intersection $\boldsymbol{m}_{\boldsymbol{k}}(i)$. We define poles $\boldsymbol{p}_{\boldsymbol{k}}$ as the centers of those segments containing at 141 least one intersection (Figure 2). Segments containing no intersection with the subnetwork are 142 disregarded. The fundamental principle of the EHM method is that all intersections contained in the 143 same segment are set to the same hydraulic head corresponding to the head of the pole. These 144 additional equalities reduce the number of unknowns at the cost of the approximation that close 
145 intersections have the same hydraulic head. The accuracy of the approximation is function of the 146 block discretization ratio $r_{\text {block }}$ defined as the block-border discretization scale $d_{\text {block }}$ normalized by 147 the block face length. The coarsest discretization corresponds to $r_{b l o c k}=100 \%$ and gives a single pole 148 by block face. It leads to a representation close to the tensor representation (Figure 2a). It is, 149 however, not equal to a tensor. First, opposite fluxes may not be equal. Second, some faces may not 150 be intersected by the network and thus may not have led to a pole. Finer discretizations, obtained for 151 decreasing $r_{\text {block }}$ values, lead to more accurate representations converging to the DFN method when 152 all poles correspond exactly to one intersetion (Figure 2b). Like in classical numerical methods, we 153 will show in section 3 that the numerical error of the EHM method decreases monotonously with the 154 block-border discretization ratio $r_{\text {block }}$, i.e. when shifting from tensor-like to DFN methods.

\section{2.2. Construction of the block-scale Equivalent Hydraulic Matrices}

156 Equivalent Hydraulic Matrix $\boldsymbol{A}_{\boldsymbol{k}}$ expresses the linear relationship between flows and heads on the 157 block border discretization. More specifically, by developing relationship (1), coefficient $\boldsymbol{A}_{\boldsymbol{k}}(i, j)$ is 158 the contribution of the head at the $j^{\text {th }}$ pole to the flow at the $i^{\text {th }}$ pole:

$$
\boldsymbol{\phi}_{\boldsymbol{k}}(i)=\sum_{j=1}^{N_{P}^{k}} \boldsymbol{A}_{\boldsymbol{k}}(i, j) \cdot \boldsymbol{H}_{\boldsymbol{k}}(j)
$$

160 where $N_{P}^{k}$ is the pole number of block $k$ and $\boldsymbol{\phi}_{\boldsymbol{k}}(i)$ and $\boldsymbol{H}_{\boldsymbol{k}}(i)$ are the flow rate and head, 161 respectively, at $i^{\text {th }}$ pole $\boldsymbol{p}_{\boldsymbol{k}}(i) \cdot \boldsymbol{A}_{\boldsymbol{k}}(i, j)$ is also equal to the flow rate computed at pole $i$ by imposing a 162 fixed head of 1 at pole $j$ and 0 at the other ones, i.e. a fixed head of 1 for the intersections overlapped 163 by the segment centered on pole $j$ and 0 for the other ones. With these boundary conditions, all 164 coefficients of column $j$ can be simultaneously determined by a single DFN simulation (Figure 3 ).

165 The construction of the full Equivalent Hydraulic Matrix requires $N_{P}^{k}-1$ simulations and not $N_{P}^{k}$, 166 since the sum of all elements from a column of $\boldsymbol{A}_{\boldsymbol{k}}$ is equal to zero because of flow conservation 
167 (Appendix A). We underline that this method does not require any modification of the fracture 168 network structure or any realignment of fractures. The approximation lies exclusively in equating 169 flows and heads at the scale of the segment of the border discretization.

\section{$170 \quad$ 2.3. Domain-scale flow simulation}

171 Solving the flow equation at the domain scale consists in imposing the continuity of heads and flow 172 rates on poles $\boldsymbol{p}_{\boldsymbol{k}}$ positioned on the block faces. External head and flow rate boundary conditions are

173 simply implemented by imposing the head in the matrix system for the fixed head values and by 174 adding a source term for the fixed flow rates on the corresponding poles, respectively.

175 We note $P$ the union of all pole points $\boldsymbol{p}_{\boldsymbol{k}}$ with the convention that poles common to two or more 176 blocks occur only once in $P . P$ is made up of $N^{\mathrm{i}}$ poles at the interface between two blocks $\left(P^{\mathrm{i}}\right)$ and of $177 N^{\mathrm{f}}$ poles at the physical limits of the domain $\left(P^{\mathrm{f}}\right)$. The total number of poles at the domain scale $N$ is 178 equal to the sum of poles of types $P^{i}$ and $P^{f}$ :

$$
N=N^{i}+N^{f} .
$$

180 With $B(j)$ as the set of blocks sharing pole $P^{i}(j)$ and with $q_{b, P^{i}(j)}$ as the flow rate at pole $P^{i}(j)$ from 181 the $b^{\text {th }}$ block of $B(j)$, flow continuity writes:

$$
\sum_{b \in B(j)} q_{b, P^{i}(j)}=0 \forall j \epsilon \llbracket 1, N^{i} \rrbracket .
$$

183 For the $N^{\mathrm{fd}}$ fixed poles at the domain limit where a Dirichlet boundary condition is applied:

$$
H^{f d}=\left(H^{f d}\right)_{0}
$$

185 For the $N^{\mathrm{fn}}$ poles on the Neumann boundary condition, the imposed flow is simply inserted in 
equation (4). Equations (1), (4) and (5) lead to a linear system of $N$ equations of the $N$ unknown 187 heads at the poles.

188 The first advantage of the EHM method compared to existing Fracture Continuum Models (FCMs)

189 is the conservation of connectivity between blocks. In fact, faces intersected by fractures contain at 190 least one pole whereas faces without intersecting fractures do not have any pole. This prevents 191 dummy additional connectivity between blocks [Jackson et al., 2002; Reeves et al., 2008]. The 192 second advantage of the EHM method is the existence of block-scale discretization parameter $r_{\text {block}}$, 193 which can be used to tune the balance between numerical efficiency and accuracy. The third 194 advantage of the method is the systematic convergence with discretization and its adjustment to all 195 kinds of 2D synthetic fracture networks as will be shown in section 3. The main drawbacks of the 196 EHM method are the necessity to perform block-scale DFN flow simulations and the specificity of 197 the domain-scale flow simulation that precludes the use of standard softwares like MODFLOW.

\section{3. Results}

\section{3.1. Fracture network types}

200 The tested networks have been chosen so that they cover a wide range of networks both above and 201 below the REV scale, with broad and narrow length and transmissivity distributions (Table 1). 202 Extreme cases of low and high variability are tested in order to assess the method in highly203 differentiated conditions. Network types include both lattice structures (Table 2.I1) and stochastic 204 complex fracture networks (Table 2.I2-4). Stochastic fracture networks are characterized by their 205 density, orientation, length and transmissivity distributions. The domain size given by the ratio of the 206 domain length to the minimal fracture length is denoted by $L$ and set to 100 . It means that the 207 fracture length distribution covers two orders of magnitude. Density is fixed by the dimensionless 208 percolation parameter $p$, equal to the sum of the square of the fracture lengths normalized by the 
209 domain area. $p$ is a direct measure of connectivity as it is very close to 5.6 at the percolation 210 threshold, whatever the other fracture network characteristics [Bour and Davy, 1997]. Three density

211 values are used for stochastic complex fracture networks and are respectively close to threshold $212(p=6)$ and at around two and three times the density at threshold ( $p=10$ and $p=20)$. For lattice 213 structures, $p$ is close to the number of fractures within the domain and has been chosen equal to 12 214 and 192 for testing methods on sparse and dense lattices, respectively. Orientations are set to $0^{\circ}$ and $21530^{\circ}$ relative to the main flow directions for the lattice structures and are uniformly distributed for the 216 complex stochastic fracture networks. For the complex stochastic fracture networks, fracture lengths 217 are power-law distributed [Bonnet et al., 2001] according to the following distribution function:

$$
p(l) \sim l^{-a}
$$

219 where $l$ is the fracture length, $a$ is the characteristic power-law length exponent and $p(l)$ the fracture 220 number of length $l$. Natural values of $a$ derived from outcrops range in the interval [2.0,3.5]. Fracture 221 transmissivity values have been chosen to be either the same for all fractures or broadly distributed according to a lognormal distribution of logarithmic standard deviation equal to 3 [Tsang et al., 223 1996]. Flow boundary conditions are classical gradient-like boundary conditions with fixed head on 224 two opposite domain faces and a constant head gradient on the orthogonal faces (Figure 1a). The 225 bottom line of Table 2 illustrates the flow distribution computed with a broad transmissivity 226 distribution and shows the strong channeling induced by the transmissivity distribution.

\section{3.2. Comparison criteria}

228 For comparing the performance of the EHM method with other existing methods, we use an accuracy criterion and a numerical memory complexity criterion. Accuracy is defined as the mean difference between the inlet and outlet flows and their reference counterparts. The reference is obtained from 
231 the direct simulation on the domain-scale discrete fracture network. By denoting $\Phi_{m}^{f_{i}}$ and $\Phi_{r e f}^{f_{i}}$ the 232 flow rates obtained respectively by the method " $m$ " and the reference method on face $f_{i}$, the 233 comparison criterion writes:

$$
\text { flow_error }_{m}=\frac{1}{2}\left(\left|\frac{\Phi_{m}^{f_{l}-\Phi_{r e f}^{f_{l}}}}{\Phi_{r e f}^{f_{l}}}\right|+\left|\frac{\Phi_{m}^{f_{r}-\Phi_{r e f}^{f_{r}}}}{\Phi_{r e f}^{f_{r}}}\right|\right) \times 100
$$

235 where $f_{l}$ and $f_{r}$ stand for the left and right vertical domain faces.

236 The memory complexity criterion is taken as the number of non-zero elements $n n z$ of matrix $B$ in the

237 linear system $B x=b$ issued from the discretization of the flow equation at the domain scale. Even if 238 the number of non-zero elements is not the ideal criterion, it is still better than the system size in this 239 case where the limitation lies rather in memory requirements than in computation time. All results 240 represent averages over 10 simulations. We have checked that for the most complex cases $D 0$ and $241 D 1,10$ and 100 simulations give very close results. Accuracy and numerical memory complexity 242 results are computed for several discretizations characterized by the number of blocks (domain-scale 243 discretization) and by $r_{\text {block }}$ (block-scale discretization).

\section{$244 \quad$ 3.3. Results with existing mapping and tensor methods}

245 To assess the Equivalent Hydraulic Matrices method, we compare it with other existing methods:

246 first with what we call the ANIS_GEO method representing permeability by a diagonal tensor 247 derived from fracture geometrical mapping onto the blocks and used within a finite volume method 248 [Botros et al., 2008] and second with what we call the TENSOR_SIM method representing 249 permeability by a full tensor obtained from block-scale DFN flow simulations and used within a 250 mixed hybrid finite element framework (Appendix B). For these two methods, the matrix 251 permeability is fixed to $10^{-12} \mathrm{~m} / \mathrm{s}$. We use these two methods only when they are strictly applicable. 
252 From [Botros et al., 2008], the ANIS_GEO method is applicable only if the ratio of the block length 253 to the minimal fracture length is lower than 2.5. For the stochastic complex networks (Table $1 \mathrm{~B} 0$ 254 D1), the ratio of the domain size to the minimal fracture length is $L=100$, requiring for the 255 ANIS_GEO method a domain-scale discretization of at least $40 \times 40$ blocks. As the TENSOR_SIM 256 method relies on the full permeability tensor at the block scale, we have determined this parameter 257 for all studied networks from the block-scale directional permeability plots (Table 3). The method is 258 applicable only when the directional permeability is close to an ellipse [Long et al., 1982]. It is the 259 case for networks $A 0, A 2$ and $D O$ (Table 3). For the other networks, transmissivity and fracture 260 length distributions display heterogeneities that cannot be represented by a tensor at the scale of the 261 block.

262 Table 4 shows the flow error as measured by (7) using the ANIS_GEO, TENSOR_SIM and EHM 263 methods for several domain discretizations. With the ANIS_GEO method, the flow error decreases 264 systematically from a $50 \times 50$ to a $200 \times 200$ domain discretization. ANIS_GEO is particularly accurate 265 for sparse flow structures (networks with a small fracture density or with a broad transmissivity 266 distribution). In fact, the simple summation of the fracture contributions induced by the mapping 267 increases sub-block-scale connectivity and hence increases flow errors. Results also show that 268 ANIS_GEO is not applicable to networks with connectivity driven by small fractures $(3<a<3.5)$, 269 yielding errors systematically larger than $41 \%$. To be applied systematically, the geometrical 270 projection method ANIS_GEO requires high levels of discretization involving large linear systems 271 (Table 5). Such discretization levels can be achieved in 2D but likely not in 3D.

272 The TENSOR_SIM method is accurate for regular and dense structures with an error lower than $1 \%$ 273 for network $A O$ (Table 4). As opposed to the ANIS_GEO method, the error decreases when the block 274 scale increases since the block becomes closer and eventually larger than the REV [Li et al., 2009]. 275 The main drawback of this method is its highly limited range of application. Most of the tested 
networks of Table 1 did not fulfill its conditions of application.

\section{$277 \quad 3.4 . \quad$ Assessment of the EHM method}

278 We have tested two levels of block-scale discretization of the EHM method: $r_{\text {block }}=10 \%$ (called the 279 most accurate method) and $r_{\text {block }}=25 \%$ (called the least accurate method). The EHM method gives 280 much smaller errors than those given by the geometrical and tensor methods ANIS_GEO and 281 TENSOR_SIM (Table 4) except for $A O$ (dense lattice structure with uniform fracture transmissivity) 282 and $D 0$ (dense fracture network with uniform fracture transmissivity) with a domain discretized by $28310 \times 10$ blocks and $r_{\text {block }}=25 \%$. For these two cases, the tensor method gives smaller errors than the 284 least accurate EHM method. In fact, the tensor method is very accurate because the REV is smaller 285 than the block. The large errors of the least accurate EHM method are linked to the large number of 286 fracture intersection points with the block border set to the same head, i.e. the head of the 287 corresponding pole. The merged points are quantified by the border merging percentage $p_{\text {border }}$ equal 288 to the difference in percentage between the intersection point and pole numbers. $p_{\text {border }}$ is $0 \%$ in the 289 absence of any approximation of the block-scale discretization and increases as larger 290 approximations are induced by the use of a smaller number of poles for the block-scale 291 discretization. For $A O$ and $D O$ with the $10 \times 10$ domain discretization and $r_{\text {block }}=25 \%$, $p_{\text {border }}$ is larger 292 than $90 \%$ and $70 \%$, respectively (Table 6). This explains the cases where the EHM method is less 293 accurate than the TENSOR_SIM method. For the same networks with finer domain discretizations 294 (30×30 and 50×50 blocks), trends are reversed and the EHM method becomes more accurate than 295 the tensor method. For lattice cases, the flow error with the EHM method is smaller than 5\% for a 296 domain discretization of $50 \times 50$ blocks.

297 For stochastic complex fracture networks, flow errors range from $0.11 \%$ to $180 \%$ with a majority of 298 errors below 10\% (Table 4). Errors larger than $10 \%$ affect cases $B 2$ and $C 2$ characterized by a coarse 
299 discretization of $10 \times 10$ blocks and by networks with the narrowest length distribution corresponding

300 to $a=3.5$. The latter fracture networks have the largest number of fractures and fracture border

301 intersections inducing first a stronger decrease in the numerical memory complexity (Table 5), and

302 then larger values of point merging percentages $p_{\text {border }}$ (Table 6). In all other cases, the flow error is 303 smaller than $5 \%$ for a domain discretization of $50 \times 50$ blocks. With the most accurate method 304 corresponding to $r_{b l o c k}=10 \%$ and a domain discretization of $50 \times 50$ blocks, errors range between $3050.11 \%$ and $2.1 \%$. For 9 out of the 12 test cases for which $\sigma_{\ln T}=3$ corresponds to a fracture 306 transmissivity distribution spanning at least 3 orders of magnitude, errors remain as low as a few 307 percents showing the very good performance of the EHM method for complex flow structures.

308 Results of Table 4 show two interesting properties of the EHM method. First, errors are not sensitive 309 to the fracture transmissivity distribution as shown by the comparison of the $D 0$ and $D 1$ cases.

310 Second, errors systematically decrease both with the domain discretization at constant $r_{b l o c k}$ and with $311 r_{\text {block }}$ at constant domain discretization for all complex stochastic fracture networks. These properties

312 offer possibilities to control the error by decreasing either the domain-scale discretization in blocks 313 or the block-scale discretization ratio $r_{\text {block}}$. We note that all the above simulations have been 314 performed on the backbone. However the applicability of the EHM method is not restricted to the 315 backbone as shown by its good performance on infinite clusters (Table 7). Even if errors increase by 316 a factor of 5 from the backbone to the infinite cluster, they still remain lower than $10 \%$ with the least 317 accurate method $\left(r_{\text {block }}=10 \%\right)$ and a domain discretization of $50 \times 50$.

\section{$318 \quad 3.5$. Flow error versus numerical memory complexity}

319 Numerical memory complexity is taken as the number of non-zero elements in the domain-scale 320 linear system issued from the discretization of the flow equation ( $n n z)$ (Table 5). $n n z$ determines the 321 memory required to solve the linear system. It does not, however, take into account the computation 
322 of the Equivalent Hydraulic Matrices at the block scale as they are not critical in terms of system size 323 and memory requirements. With the classical ANIS_GEO and TENSOR_SIM methods, the 324 numerical memory complexity increases quadratically with the discretization ratio. With the EHM 325 method, the numerical memory complexity is more variable and increases more slowly. Whatever 326 the domain discretization and the value of $r_{\text {block }}$ for complex stochastic fracture networks, EHM 327 methods yield smaller numerical memory complexity than the DFN method except for the $B 0$ case. 328 In the latter case, the proportion of blocks crossed by a single fracture increases the numerical 329 memory complexity without improving the accuracy.

330 A more advanced evaluation of the methods is proposed by comparing their error according to their 331 numerical memory complexity (Figures 4-6). For lattice structures (Figure 4 except magenta 332 symbols), the EHM method is orders of magnitude more accurate than the classical methods at 333 comparable complexities except for the $A O$ case already discussed in section 3.4. Figure 4 also shows 334 that the accuracy of the TENSOR_SIM method increases with the numerical memory complexity as 335 discussed in section 3.3. For the dense complex stochastic fracture network of case $D 0$ (Figure 4, 336 magenta symbols), the error with the TENSOR_SIM method is smaller than the error with all other 337 methods at very low complexity (11\%) but cannot be made smaller by refining the discretization. By 338 contrast, with the EHM method, the error is larger at small complexity but decreases to less than $1 \%$ 339 for the highest complexities. For the stochastic complex fracture networks (Figures 5-6), errors with 340 the EHM method decrease with the numerical memory complexity (nnz), with a systematic trend 341 close to $n n z^{-1}$. Figures 4-6 show that the errors using the EHM method with $r_{\text {block }}=10 \%$ and $r_{\text {block }}$ $342=25 \%$ are roughly parallel in log-log plots. For the same level of error corresponding to horizontal 343 lines in Figures 4-6, the $r_{\text {block }}=10 \%$ method yields smaller numerical memory complexities than the 344 method with $r_{\text {block }}=25 \%$.

\section{3.6. Parameter optimization}


346 The choice of the optimal method parameters depends on the targeted accuracy, available 347 computation time and memory and on the fracture network structure. We illustrate the methodology 348 to determine the appropriate parameter values on the most complex fracture network presented 349 before $D 1$. Basically, we show in this section that the accuracy is controlled by the discretization 350 ratio $r_{\text {block }}$ times the length of the block edge while computation time and memory requirements are 351 controlled by the inverse of the discretization ratio $\left(1 / r_{\text {block }}\right)$. The approximation of the method is 352 performed on the block-border discretization by equating the head of points belonging to the same 353 discretization segment. The sole parameter influencing accuracy is thus the normalized segment 354 length $d_{\text {block }}$ equal to the discretization ratio $r_{\text {block }}$ times the length of the block edge divided by the 355 minimal fracture length. The error error_flow defined in (7) increases monotonously with $d_{b l o c k}$ 356 (Figure 7). Flow errors smaller than $20 \%$ are obtained for $d_{\text {block }}$ values smaller than 2 . Once the 357 segment length has been fixed by the targeted accuracy, the computation time and memory 358 requirements are adjusted by choosing the discretization of the system in blocks controlled by the 359 parameter $1 / r_{\text {block }}$ (Figure 8 ). Here the computation time refers to the full time of the flow simulation 360 including the determination of the Equivalent Hydraulic Matrices and the solution of the large 361 system issued by the domain-scale flow discretization. Memory requirements are still taken as the 362 number of non-zero elements in the domain-scale matrix ( $n n z)$. As previously said, $n n z$ decreases for 363 coarser domain discretizations. The computation is mainly controlled by the determination of the 364 Equivalent Hydraulic Matrices. It first sharply decreases with $1 / r_{\text {block }}$ and then increases slightly. The 365 minimum expresses an optimal distribution of computations between the domain scale and the block 366 scale. Smaller $1 / r_{\text {block }}$ values yield more numerous smaller blocks and more Equivalent Hydraulic 367 Matrices to determine and in turn an increase of the full computation time by more than order of 368 magnitude. Large $1 / r_{\text {block }}$ values yield less numerous larger blocks which Equivalent Hydraulic 369 Matrices take a much larger time to determine, increasing the full computation time by at least $50 \%$. 
370 Similar results showing the existence of the minimum have been obtained for greater number of

371 Monte-Carlo simulations and for different fracture network structures.

\section{4. Discussion}

373 The principle of the Equivalent Hydraulic Matrices method is to distribute the numerical complexity 374 among two scales, the block-scale and the domain-scale. This method introduces a reduction of the 375 domain-scale numerical memory complexity by coarsening the block-border discretization. The 376 approximation consists in equating heads on nearby network points. It remains local and adjusts 377 automatically to the specific network configuration. Like the tensor and geometrical mapping 378 methods, the EHM method increases connectivity along block interfaces but only through the 379 introduction of shortcuts between existing paths and not through the connection of otherwise 380 disconnected faces. Moreover, the connectivity increase is limited to the block borders and does not 381 affect the connectivity within the block.

382 The EHM method is structured around the block-scale Equivalent Hydraulic Matrices, which transfer 383 the local connectivity information from the block scale to the domain scale. The Equivalent 384 Hydraulic Matrices are determined by the configurations of the fracture network within the blocks 385 but do not depend on the boundary conditions. In other words, the matrices are not intrinsic medium 386 properties like a tensor but can be used instead of the discrete fracture network in all flow contexts 387 both above and below the Representative Elementary Volume (REV). The Equivalent Hydraulic 388 Matrices method is still applicable below the REV due to the adjustment of the block-scale matrices 389 to the specificity of the connectivity structures.

390 Because the Equivalent Hydraulic Matrices are derived from DFN computations, it is not surprising 391 that they contain more information than the geometrical projection methods and lead to better performance at equivalent domain-scale numerical memory complexity. We express the domain- 
393 scale numerical memory complexity by the number of non-zero elements $(n n z)$ of the linear system 394 issued from the discretization of the flow equation. $n n z$ is two to four orders magnitude smaller with 395 the EHM method than with geometrical projection methods. The EHM method also displays 396 systematically decreasing flow errors with the domain discretization and block-scale discretization 397 parameter $r_{\text {block}}$. This offers possibilities to find the best optimal complexity for a given error 398 requirement. As seen in section 3.3, this is not possible with the tensor method TENSOR_SIM and it 399 requires too fine a domain discretization with the geometrical method ANIS_GEO.

400 The EHM method is intermediary between the full DFN flow simulation and the tensor method. Like 401 in the classical tensor methods [Jackson et al., 2002], the method relies on block-scale DFN 402 simulations. It is also similar to classical numerical methods from several respects. First, it expresses 403 the relationship between flows and heads on the block borders like many numerical methods such as 404 finite element or boundary element methods. Second, it converges to the full DFN solution when the 405 domain discretization or the block-scale discretization increases. As a two-scale method, it shares 406 similarities with multiscale methods like multigrid methods. It is, however, a pure bottom-up 407 approach in the sense that the block-scale information is used at domain scale but not the other way 408 around. From this respect, it is closer to the principle of the multiscale finite element methods 409 [Efendiev and Hou, 2007] than to the principle of multigrid methods [Wesseling, 2004]. Finally, it 410 remains opposed to homogenization methods since the Equivalent Hydraulic Matrices strongly 411 depend on the block-scale fracture network structure and cannot be extrapolated to other blocks or 412 other scales.

413 However, EHM methods have two drawbacks, the first one being the specificity of the domain-scale 414 simulation method that precludes the use of commonly available continuous flow simulation 415 softwares like MODFLOW. The second drawback is the additional numerical time complexity 416 arising from the computation of the block-scale equivalent matrices. The total numerical complexity 
417 includes the solution of the domain-scale linear system and the computation of the Equivalent

418 Hydraulic Matrices at the block scale. The first contribution is evaluated by the number of non-zero

419 elements in the domain-scale linear system $n n z$ used in the previous section. The second contribution

420 is a function of the number of block-scale simulations multiplied by the complexity of the block-

421 scale simulations. We have chosen to retain only the first contribution to the numerical complexity

422 for the two following reasons. First, the complexity of the domain-scale linear system is a critical 423 constraint. Very large systems corresponding to $n n z>10^{7}$ require parallel computation. While this 424 constraint is met only for very large systems in 2D, it is current for 3D fracture networks at much 425 smaller domain scales. Second, the EHM methods will likely be interesting for transient simulations. 426 In fact, the computation of the EHMs will be performed only once and the complexity of the 427 transient simulations will depend only on the domain-scale linear system complexity. The choice of 428 both the domain discretization and the block-scale discretization parameter will be dictated by the 429 numerical optimization, the performance of simulations through block-scale and domain-scale 430 computations restricted to manageable sizes, and last but not least by the required accuracy.

\section{5. Conclusion}

432 We have presented a new mapping method for solving the flow equation in 2D discrete fracture 433 networks. The method consists in superposing a mesh onto the fracture network and finding the 434 relationship between heads and flows on the borders of each block of the mesh. The relationship is 435 linear and can be expressed in matrix form, hence the name the "Equivalent Hydraulic Matrices" 436 (EHM) method. We have shown that this linear relationship is fundamentally analog to Darcy's law 437 as it is equivalent to relating flows to well-chosen head gradients on block borders. The matrix 438 coefficients can be determined by block-scale numerical simulations and express equivalent block439 scale permeability between block border zones. The zones are chosen independently for each block 440 interface and correspond to the discretization of intersection points between the fracture network and 
441 the block border. The method is parameterized both by the block-scale discretization parameter 442 (block-scale discretization distance divided by the characteristic block scale) and the domain 443 discretization (the domain scale divided by characteristic block scale in each direction). The flow 444 simulation at the domain scale is performed simply by assembling the block-scale Equivalent 445 Hydraulic Matrices through head and flow continuity conditions.

446 The interest of the EHM method is to keep good approximations of both the internal block and inter447 block connectivities. Discretization is performed at a local scale and adjusts automatically to local 448 fracture network configurations. We show on a broad range of 2D fracture networks with different 449 density, fracture length and transmissivity distributions that the relative error of the method decreases 450 systematically with the domain discretization and the block-scale discretization parameter, allowing 451 for a possible automatic control of the method accuracy. We also show that the relative error of the 452 EHM method remains restricted to a few percents for a coarse domain discretization $(30 \times 30$ to 453 50×50), whatever the network geometrical structure and the fracture transmissivity distribution. The 454 main advantage is its applicability to all kind of network structures, whereas the tensor method can 455 only be used for blocks larger than the Representative Elementary Volume, a too restrictive 456 condition for general DFN simulations. Geometrical methods give results of comparable accuracy 457 for a much larger domain discretization leading to domain-scale numerical memory complexities 458 orders of magnitude larger than the numerical memory complexity of the EHM method. The EHM 459 method enables large-scale 2D flow simulation networks. We intend to test its performance on 3D 460 fracture network simulations and in transient flow contexts. 


\section{Appendices}

462 Appendix A: Property of the Equivalent Hydraulic Matrix

463 With the construction method described in section 2.2, $\boldsymbol{A}_{\boldsymbol{k}}$ has several properties. First, by imposing 464 a fixed head of 1 at pole $j$ and 0 at the other ones as boundary conditions, the flow goes into the 465 block by $\boldsymbol{p}_{\boldsymbol{k}}(j)$ and outward through the other poles $\boldsymbol{p}_{\boldsymbol{k}}(i)(i \neq j)$. Considering the flow going into 466 the block as positive and the flow going outward as negative leads to:

$$
\left\{\begin{array}{c}
\boldsymbol{A}_{\boldsymbol{k}}(j, j) \geq 0 \\
\boldsymbol{A}_{\boldsymbol{k}}(i, j) \leq 0, i \neq j
\end{array}\right.
$$

468 Second, for a given column $j$, all elements $\boldsymbol{A}_{\boldsymbol{k}}(i, j)$ are determined simultaneously by solving the 469 flow equation; mass conservation implies that

$$
\sum_{i=1}^{N_{P}^{k}} \boldsymbol{A}_{\boldsymbol{k}}(i, j)=0 .
$$

471 Or similarly:

$$
\boldsymbol{A}_{\boldsymbol{k}}(i, i)=-\sum_{j=1, j \neq i}^{N_{P}^{k}} \boldsymbol{A}_{\boldsymbol{k}}(j, i) .
$$

473 Third, because the reciprocity principle is applicable in the case of Darcian flow [Barker, 1991], $\boldsymbol{A}_{\boldsymbol{k}}$ 474 is symmetric:

$$
\boldsymbol{A}_{\boldsymbol{k}}(i, j)=\boldsymbol{A}_{\boldsymbol{k}}(j, i)
$$

476 Fourth, we show that the linear relationship (1) between flows and heads with property (10) leads to 477 a relationship between flows and head gradients. In fact: 


$$
\begin{gathered}
\boldsymbol{\phi}_{\boldsymbol{k}}(i)=\sum_{j=1}^{N_{P}^{k}} \boldsymbol{A}_{\boldsymbol{k}}(i, j) \times \boldsymbol{H}_{\boldsymbol{k}}(j) \\
\boldsymbol{\phi}_{\boldsymbol{k}}(i)=\sum_{j=1, j \neq i}^{N_{P}^{k}} \boldsymbol{A}_{\boldsymbol{k}}(i, j) \times \boldsymbol{H}_{\boldsymbol{k}}(j)+\boldsymbol{A}_{\boldsymbol{k}}(i, i) \times \boldsymbol{H}_{\boldsymbol{k}}(i)
\end{gathered}
$$

478 and using (10):

$$
\boldsymbol{\phi}_{\boldsymbol{k}}(i)=\sum_{j=1, j \neq i}^{N_{P}^{k}} \boldsymbol{A}_{\boldsymbol{k}}(i, j) \times\left(\boldsymbol{H}_{\boldsymbol{k}}(j)-\boldsymbol{H}_{\boldsymbol{k}}(i)\right)
$$

$$
\boldsymbol{\phi}_{\boldsymbol{k}}(i)=\sum_{j=1}^{N_{P}^{k}} \boldsymbol{A}_{\boldsymbol{k}}(i, j) \times x_{k, i j} \times \frac{\left(\boldsymbol{H}_{\boldsymbol{k}}(j)-\boldsymbol{H}_{\boldsymbol{k}}(i)\right)}{x_{k, i j}}
$$

480 where $x_{k, i j}$ is the distance between poles $\boldsymbol{p}_{\boldsymbol{k}}(i)$ and $\boldsymbol{p}_{\boldsymbol{k}}(j)$. Equation (12) shows that flow $\boldsymbol{\phi}_{\boldsymbol{k}}(i)$ at $481 \boldsymbol{p}_{\boldsymbol{k}}(i)$ is the sum of the head gradients from $\boldsymbol{p}_{\boldsymbol{k}}(i)$ to the other poles. Equation (12) gives a simple 482 interpretation of $\boldsymbol{A}_{\boldsymbol{k}}(i, j) \times x_{k, i j} . \boldsymbol{A}_{\boldsymbol{k}}(i, j) \times x_{k, i j}$ is the proportionality coefficient between flow $483 \boldsymbol{\phi}_{\boldsymbol{k}}(i)$ and the head gradient $\left(\boldsymbol{H}_{\boldsymbol{k}}(j)-\boldsymbol{H}_{\boldsymbol{k}}(i)\right) / x_{k, i j}$ between $\boldsymbol{p}_{\boldsymbol{k}}(i)$ and $\boldsymbol{p}_{\boldsymbol{k}}(j) \cdot \boldsymbol{A}_{\boldsymbol{k}}(i, j) \times x_{k, i j}$ can 484 thus be interpreted as an "equivalent transmissivity" between the $i^{\text {th }}$ and $j^{\text {th }}$ poles. 
487 The Equivalent Hydraulic Matrices method consists in dividing the domain into blocks and 488 describing block-scale hydraulic properties using Equivalent Hydraulic Matrices. The discretization 489 of the block borders by poles $\boldsymbol{p}_{\boldsymbol{k}}$ is determined by the block-scale discretization parameter $r_{b l o c k}$, 490 which is the ratio of the block-scale distance discretization to the block length. This parameter $r_{\text {block }}$ 491 drives the discretization of intersections $\boldsymbol{m}_{\boldsymbol{k}}$ between block borders and fractures. Coefficients of the 492 EHMs are determined by simulations at the block scale as described in section 2.2. The EHMs are 493 equivalent to tensors in that they impose the following discretization and construction rules: (1) $494 r_{\text {block }}=100 \%$, i.e. each block border is discretized by at most one pole, (2) each block border is 495 represented by one pole (even if there is no intersection point), (3) matrix coefficients are determined 496 by applying head gradient boundary conditions in the vertical and horizontal directions [Renard et 497 al., 2001], (4) the computed flow rates used for the determination of the coefficients are the 498 directional flow rates, i.e. the mean of the flow rates going out of the domain through borders 499 perpendicular to the studied direction, and (5) coefficients are corrected to obtain symmetric positive 500 definite tensors [Long et al., 1982]. Adding these rules of determination, the Equivalent Hydraulic 501 Matrices become tensors that describe block-scale permeability. Computed block-scale tensors are 502 used within a classical mixed hybrid method adapted for quadrangles to simulate flow at the domain 503 scale [Chavent and Roberts, 1991]. We denote this method the TENSOR_SIM method. 
$506 K_{x x} \quad$ permeability in the $x$-direction due to a head gradient in the $x$-direction, $\mathrm{m} / \mathrm{s}$.

$507 \quad K_{y y} \quad$ permeability in the $y$-direction due to a head gradient in the $y$-direction, $\mathrm{m} / \mathrm{s}$.

$508 K_{x y} \quad$ permeability in the $x$-direction due to a head gradient in the $y$-direction, $\mathrm{m} / \mathrm{s}$.

$509 K_{y x} \quad$ permeability in the $y$-direction due to a head gradient in the $x$-direction, $\mathrm{m} / \mathrm{s}$.

$510 \quad \boldsymbol{p}_{\boldsymbol{k}} \quad$ vector of poles.

$511 \boldsymbol{\Phi}_{\boldsymbol{k}} \quad$ vector of flow rates at the poles for block $k, \mathrm{~m}^{2} / \mathrm{s}$.

$512 \boldsymbol{H}_{\boldsymbol{k}} \quad$ vector of heads at the poles for block $k, \mathrm{~m}$.

$513 \quad \boldsymbol{A}_{\boldsymbol{k}} \quad$ Equivalent Hydraulic Matrix of block $k, \mathrm{~m} / \mathrm{s}$.

$514 \boldsymbol{m}_{\boldsymbol{k}} \quad$ vector of intersections between the fractures and the faces of block $k$.

$515 \quad d_{\text {block }} \quad$ discretization distance of block borders, $\mathrm{m}$.

$516 \quad r_{\text {block }} \quad$ discretization ratio of block borders.

$517 \quad N_{P}^{k} \quad$ number of poles of block $k$.

$518 x_{k, i j} \quad$ distance between the $i^{\text {th }}$ and $j^{\text {th }}$ poles, $\mathrm{m}$.

$519 P \quad$ union of all poles. 
$520 \quad P^{i}$

$521 \quad P^{f}$

$522 N$

$523 \quad N^{\mathrm{i}}$

$524 \quad N^{\mathrm{f}}$

$525 \quad B(j)$

$526 q_{b, P^{i}(j)}$

$527 \quad N^{\mathrm{fd}}$

$528 \quad N^{\mathrm{fn}}$

$529 H^{f d}$

$530 \quad\left(H^{f d}\right)_{0}$

$531 p$

$532 l$

$533 p(l)$

$534 a$ union of poles on block interfaces.

union of poles on domain faces.

total number of poles.

number of poles of type $P^{\mathrm{i}}$.

number of poles of type $P^{\mathrm{f}}$.

set of blocks sharing pole $P^{i}(j)$

flow rate at poles $P^{i}(j)$ from the $b^{\text {th }}$ block, $\mathrm{m}^{2} / \mathrm{s}$.

number of poles on the Dirichlet boundary condition.

number of poles on the Neumann boundary condition.

head of poles on the Neumann boundary condition, $m$

fixed head on the Neumann boundary condition, $m$

percolation parameter.

fracture length, $\mathrm{m}$.

fracture length distribution.

power law exponent. 
$535 \Phi_{m}^{f_{i}} \quad$ flow rate computed by the method " $m$ " on the face $f_{i}, \mathrm{~m}^{2} / \mathrm{s}$.

$536 \quad \Phi_{r e f}^{f_{i}} \quad$ flow rate computed by the reference method on the face $f_{i}, \mathrm{~m}^{2} / \mathrm{s}$.

$537 \quad n n z \quad$ number of non-zero elements of the domain-scale linear system.

$538 \quad p_{\text {border }} \quad$ border discretization percentage 


\section{Acknowledgments}

540 This work was supported by the ANR project MICAS. We thank Jocelyne Erhel for fruitful 541 discussions. 
543 Ando, K., et al. (2003), Stochastic continuum modeling of flow and transport in a crystalline rock 544 mass: Fanay-Augères, France, revisited, Hydrogeology Journal, 11(5).

545 Baghbanan, A., and L. R. Jing (2007), Hydraulic properties of fractured rock masses with correlated 546 fracture length and aperture, International Journal of Rock Mechanics and Mining Sciences, 44(5), $547 \quad 704-719$.

548 Barker, J. A. (1991), The reciprocity principle and an analytical solution for darcian flow in a 549 network, Water Resour. Res., 27(5), 743-746.

550 Berkowitz, B., et al. (2000), Scaling of fracture connectivity in geological formations, Geophys. Res. 551 Lett., 27(14), 2061-2064.

552 Bonnet, E., et al. (2001), Scaling of Fracture Systems in Geological Media, Reviews of Geophysics, 553 39(3), 347-383.

554 Botros, F. E., et al. (2008), On mapping fracture networks onto continuum, Water Resour. Res., $55544(8)$.

556 Bour, O., and P. Davy (1997), Connectivity of random fault networks following a power law fault 557 length distribution, Water Resources Research, 33(7), 1567-1583.

558 Bour, O., and P. Davy (1998), On the connectivity of three dimensional fault networks, Water 559 Resources Research, 34(10), 2611-2622.

560 Bourbiaux, B., et al. (1998), A rapid and efficient methodology to convert fractured reservoir images 561 into a dual-porosity model, Rev. Inst. Fr. Pet., 53(6), 785-799.

562 Chavent, G., and J. E. Roberts (1991), A unified physical presentation of mixed, mixed-hybrid finite563 elements and standard finite-difference approximations for the determination of velocities in 564 waterflow problems, Advances in Water Resources, 14(6), 329-348.

565 Cvetkovic, V., et al. (2004), Stochastic simulation of radionuclide migration in discretely fractured 566 rock near the Äspö Hard Rock Laboratory, Water Resources Research.

567 Davis, T. A. (2004), Algorithm 832: UMFPACK V4.3---an unsymmetric-pattern multifrontal 568 method, ACM Trans. Math. Softw., 30(2), 196-199.

569 Davy, P., et al. (2006), Flow in multiscale fractal fracture networks, Fractal Analysis for Natural 570 Hazards(261), 31-45.

571 Davy, P., et al. (2009), A Universal Model of Fracture Scaling and its consequence for crustal hydro572 mechanics, Journal of Geophysical Research, submitted. 
573 de Dreuzy, J. R., et al. (2001a), Hydraulic properties of two-dimensional random fracture networks 574 following a power law length distribution: 2-Permeability of networks based on log-normal 575 distribution of apertures, Water Resources Research, 37(8), 2079-2095.

576 de Dreuzy, J. R., et al. (2001b), Hydraulic properties of two-dimensional random fracture networks 577 following a power law length distribution 1. Effective connectivity, Water Resour. Res., 37(8), 20655782078.

579 de Dreuzy, J. R., et al. (2002), Permeability of 2D fracture networks with power-law distributions of $580 \quad$ length and aperture, Water Resources Research, 38(12).

581 de Dreuzy, J. R., and J. Erhel (2002), Efficient algorithms for the determination of the connected 582 fracture network and the solution of the steady-state flow equation in fracture networks, Computers 583 and Geosciences, 29(107-111).

584 Dershowitz, W. S., and C. Fidelibus (1999), Derivation of equivalent pipe network analogues for 585 three-dimensional discrete fracture networks by the boundary element method, Water Resour. Res., 586 35(9), 2685-2691.

587 Durlofsky, L. J. (1991), Numerical calculation of equivalent grid block permeability tensors for 588 heterogeneous porous media, Water Resour. Res., 27(5), 699-708.

589 Efendiev, Y., and T. Hou (2007), Multiscale finite element methods for porous media flows and their 590 applications, Applied Numerical Mathematics, 57(5-7), 577-596.

591 Erhel, J., et al. (2009), A parallel scientific software for heterogeneous hydrogeoloy, in Parallel 592 Computational Fluid Dynamics 2007, edited, pp. 39-48.

593 Falgout, R. D., et al. (2005), Pursuing scalability for Hypre's conceptual interfaces, ACM Trans. 594 Math. Softw., 31(3), 326-350.

595 Hsieh, P. A. (1998), Scale effects in fluid flow through fractured geological media, in Scale 596 dependence and scale invariance in hydrology, edited, pp. 335-353, Cambridge University Press.

597 Jackson, C. P., et al. (2002), Self-consistency of a heterogeneous continuum porous medium 598 representation of a fractured medium, Water Resour. Res., 36.

599 Karimi-Fard, M., et al. (2006), Generation of coarse-scale continuum flow models from detailed 600 fracture characterizations, Water Resources Research, 42(10).

601 Lee, S. H., et al. (2001), Hierarchical modeling of flow in naturally fractured formations with 602 multiple length scales, Water Resources Research, 37(3), 443-455.

$603 \mathrm{Li}, \mathrm{J} . \mathrm{H}$. , et al. (2009), Permeability tensor and representative elementary volume of saturated 604 cracked soil, Can. Geotech. J., 46(8), 928-942. 
605 Long, J. C. S., et al. (1982), Porous media equivalents for networks of discontinuous fractures, Water 606 Resour. Res., 18(3), 645-658.

607 Neuman, S. P. (2005), Trends, prospects and challenges in quantifying flow and transport through 608 fractured rocks, Hydrogeology Journal, 13(1), 124-147.

609 Reeves, D. M., et al. (2008), Transport of conservative solutes in simulated fracture networks: 1. 610 Synthetic data generation, Water Resour. Res., 44(5).

611 Renard, P., et al. (2001), Laboratory determination of the full permeability tensor, Journal of 612 Geophysical Research, 106(B11), 26443-26452.

613 Svensson, U. (2001), A continuum representation of fracture networks. Part I: Method and basic test 614 cases, Journal of Hydrology, 250(1-4), 170-186.

615 Tsang, Y. W., et al. (1996), Tracer transport in a stochastic continuum model of fractured media, 616 Water Resources Research, 32(10), 3077-3092.

617 Wesseling, P. (2004), An Introduction to Multigrid Methods, Edwards.

618

619

620 


\section{Figure captions}

623 Figure 1 - Fracture network at the block scale (a) and corresponding flows (b) for the gradient head

624 boundary conditions illustrated in (a). Fracture network parameters are the system size $L$ normalized

625 by the smallest fracture length $(L=100)$, the fracture density number twice larger as its value at 626 percolation threshold, the power-law fracture length exponent of 2.5 and the lognormal 627 transmissivity distribution of logarithmic standard deviation 3.0. Boundary flows integrated on the 628 domain sides and normalized by the mean fracture transmissivity are given in (b). They display large 629 differences between opposite sides and illustrate the non-tensor nature of the flows.

630 Figure 2 - Principle of the block-border discretization with two different discretization scales $d_{\text {block }}$ 631 corresponding to the side length (a) and to half of it (b). The backbone of the sub-network contained 632 in the block is represented by the grey segments. Intersections $\boldsymbol{m}_{\mathrm{k}}$ between the backbone and the 633 block borders are the black dots. Discretization segments and poles $\boldsymbol{p}_{\boldsymbol{k}}$ are respectively the color 634 dashed segments and crosses. In (a), the four discretization segments intersect the backbone in one or 635 two points. The four poles corresponding to the four crosses are thus defined and the Equivalent 636 Hydraulic Matrix (EHM) is of rank 4. In (b), only six of the eight discretization segments intersect 637 the backbone leading to the definition of 6 poles and to an EHM of rank 6.

638 Figure 3 - Principle of the determination of one of the columns of the Equivalent Hydraulic Matrix $639 \boldsymbol{A}_{\boldsymbol{k}}$. In this example, block $k$ is made up of fives intersections between the sub-network and the block 640 borders (black points) and four poles (blue crosses). The boundary conditions applied to poles 641 illustrated in (a) are a fixed head of 1 for the $2^{\text {nd }}$ pole and 0 for the other ones. They condition the 642 boundary conditions applied to the intersections illustrated in (b), which are a fixed head of 1 for the 
643 intersections represented by the $2^{\text {nd }}$ pole and 0 for the other ones. Flow rates in poles (d) are deduced

644 from flow rates at the intersections (c). The flow rate at the $i^{\text {th }}$ pole is the sum of the flow rates at the 645 intersections represented by this pole. The elements of the second column of the matrix $\boldsymbol{A}_{\boldsymbol{k}}$ are 646 deduced from flow rates computed at the poles (e).

647 Figure 4 - flow_error versus numerical memory complexity (nnz) for lattice structures and dense 648 fracture networks with constant fracture transmissivity (magenta symbols). The grey area underlines 649 a lower part of the graph where errors range between $5 \times 10^{-6} \%$ and $10^{-4} \%$. The dashed horizontal line 650 pictures the $10 \%$ error value. The dashed diagonal lines are power-law functions of exponent -1 and 651 are meant as a guide for the eye for the decrease tendency of the EHM method. Note that errors 652 larger than $10^{3}$ are not represented.

653 Figure 5 - flow_error versus numerical memory complexity (nnz) for stochastic complex fracture 654 networks at threshold with distributed fracture transmissivities. The dashed horizontal line pictures 655 the $10 \%$ error value. The dashed diagonal lines are power-law functions of exponent -1 and are 656 meant as a guide for the eye for the decrease tendency of the EHM method. Note that errors larger 657 than $10^{3}$ are not represented.

658 Figure 6 - flow_error versus numerical memory complexity (nnz) for stochastic complex fracture 659 networks with distributed fracture transmissivities. The dashed horizontal line pictures the $10 \%$ error 660 value. The dashed diagonal lines are power-law functions of exponent -1 and are meant as a guide 661 for the eye for the decrease tendency of the EHM method. Note that errors larger than $10^{3}$ are not 662 represented.

663 Figure 7 -flow_error versus $d_{\text {block }}$ the discretization ratio $r_{\text {block }}$ times the length of the block edge for 664 the network $D 1$ (domain size $L=100$ ). 
665 Figure 8 - Computation time (red dashed line) and numerical memory complexity taken as the 666 number of non-zero elements in the largest matrix (black line) as a function of block size divided by 667 the segment discretization length $1 / r_{\text {block }}$ for $D 1$ with $d_{\text {block }}$ equal to 1. 


\begin{tabular}{|c|c|c|c|c|c|}
\hline Network type & \multicolumn{4}{|c|}{ Parameters } & Table 2 \\
\hline & $p$ & $a$ & $\theta$ & $\sigma_{\ln T}$ & \\
\hline$A 0$ & 192 & 1 & $0^{\circ}, 30^{\circ}$ & 0 & \\
\hline$A 1$ & 192 & 1 & $0^{\circ}, 30^{\circ}$ & 3 & \\
\hline$A 2$ & 12 & 1 & $0^{\circ}, 30^{\circ}$ & 0 & \\
\hline$A 3$ & 12 & 1 & $0^{\circ}, 30^{\circ}$ & 3 & I1 \\
\hline$B 0$ & 6 & 2.5 & Uniform & 3 & \\
\hline$B 1$ & 6 & 3 & Uniform & 3 & $\mathrm{I} 2$ \\
\hline$B 2$ & 6 & 3.5 & Uniform & 3 & \\
\hline$\overline{C O}$ & 10 & 2.5 & Uniform & 3 & \\
\hline$C 1$ & 10 & 3 & Uniform & 3 & \\
\hline$\overline{C 2}$ & 10 & 3.5 & Uniform & 3 & I3 \\
\hline DO & 20 & 2.5 & Uniform & 0 & \\
\hline$D 1$ & 20 & 2.5 & Uniform & 3 & $\mathrm{I} 4$ \\
\hline
\end{tabular}

Table 1 - Characteristics of the tested fracture networks. The first four networks are on-lattice structures whereas the other ones are off-lattice structures. The ratio of the domain size $L$ to the length of the smallest fracture $l_{\min }$ is set to 100 . The fracture density is characterized by the percolation parameter $p$ [Bour and Davy, 1998]. Fractures either cross the whole domain $(a=1)$ or are distributed according to a power-law distribution $(a>1)$. Fracture orientations $(\theta)$ are either specified to a set of fixed angles (first four cases) or uniformly distributed. Fracture transmissivity is constant $\left(\sigma_{\ln T}=0\right)$ or lognormally distributed with a lognormal standard deviation $\left(\sigma_{\ln T}=3\right)$. The last column indicates the figure numbers displaying an example of the network type in Table 2. Fracture network types are classified in family of networks: "A" is for lattice structures, " $B$ ” for networks at percolation threshold, “ $C$ ” for networks with an intermediary fracture density and " $D$ ” for dense networks. 


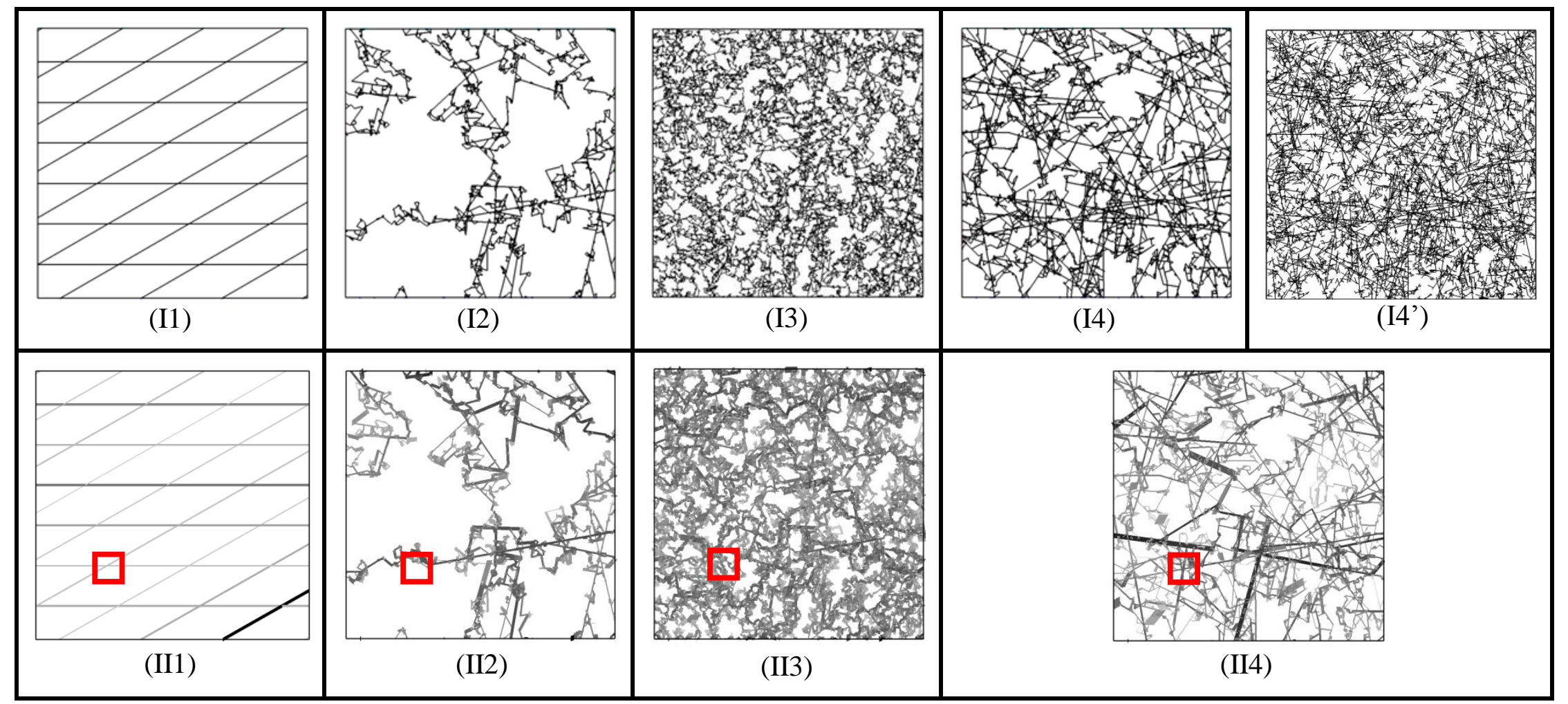

678 Table 2 - Illustration of the tested fracture networks with examples of backbones (I1-I4), infinite clusters (I4') and flows (II1-II4) obtained with

679 the gradient boundary conditions illustrated by Figure $1 \mathrm{a}$ and computed with a broadly distributed fracture transmissivity $\sigma_{\ln T}=3$ (see Table 1 ).

680 Flow magnitude is represented by grey intensity and segment width. I1, I2, I3 and I4-4' correspond to network types $A 3, B 1, C 2$ and $D 1$ (Table

681 1). Red squares stand for an elementary block corresponding to a domain discretization of $10 \times 10$ blocks. 


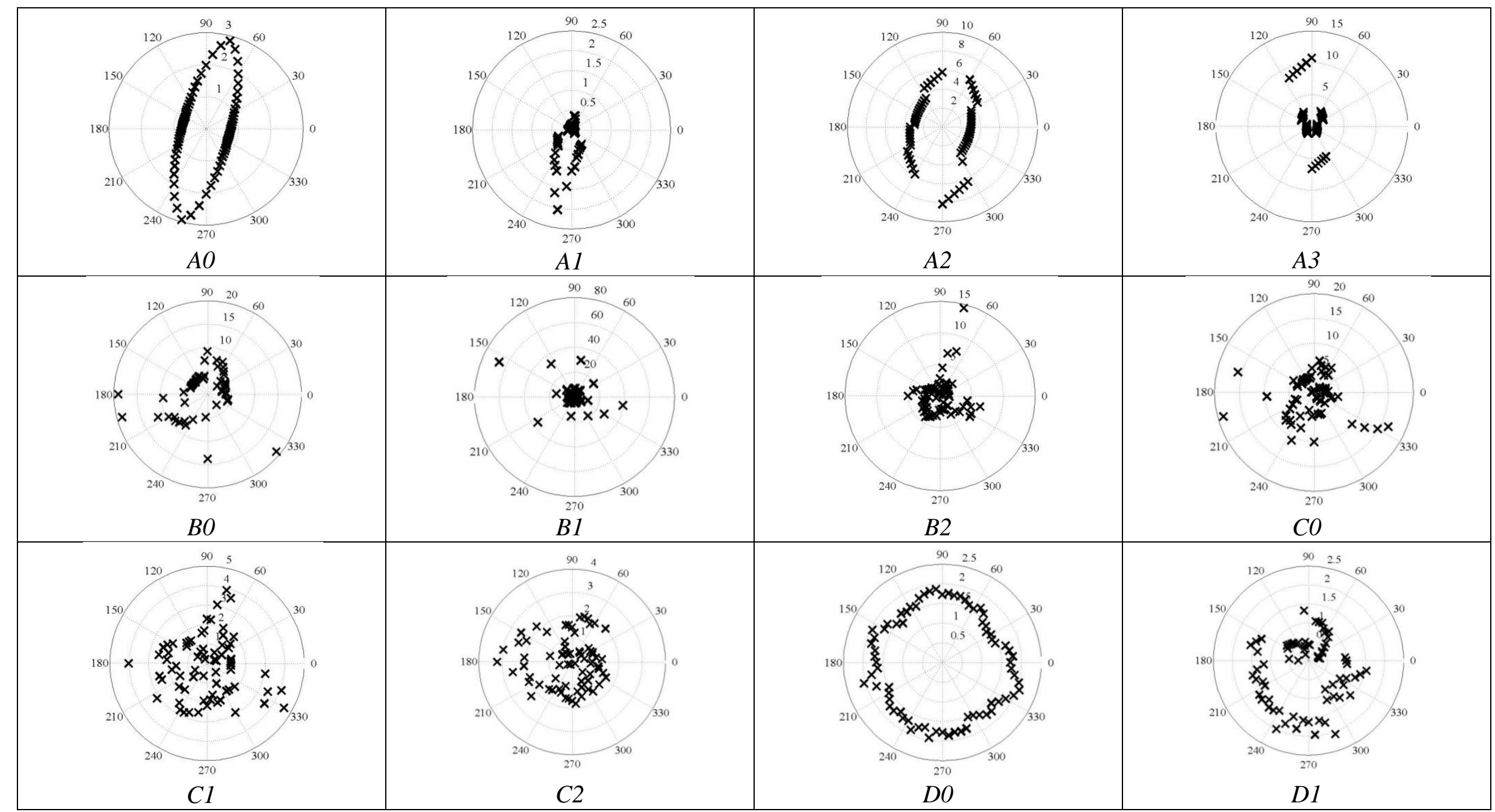

Table 3 - Polar plots of permeability for the networks of Table 1, representing the permeability versus the polar angle $\theta$. Each point represents initial system center. 


\begin{tabular}{|c|c|c|c|c|c|c|c|c|c|c|c|c|c|}
\hline \multirow[b]{2}{*}{ Domain discretization } & \multicolumn{4}{|c|}{ ANIS_GEO } & \multicolumn{3}{|c|}{ TENSOR_SIM } & \multicolumn{3}{|c|}{ EHM (10\%) } & \multicolumn{3}{|c|}{ EHM (25\%) } \\
\hline & $10 \times 10$ & $30 \times 30$ & $50 \times 50$ & $200 \times 200$ & $10 \times 10$ & $30 \times 30$ & $50 \times 50$ & $10 \times 10$ & $30 \times 30$ & $50 \times 50$ & $10 \times 10$ & $30 \times 30$ & $50 \times 50$ \\
\hline$A 0$ & 33 & 33 & 31 & 20 & 0.14 & 0.04 & 0.92 & $5.5 \times 10^{-5}$ & $2.0 \times 10^{-5}$ & $2.7 \times 10^{-5}$ & 33 & $2.3 \times 10^{-5}$ & $2.8 \times 10^{-5}$ \\
\hline$A 1$ & 44 & 49 & 47 & 2.3 & & & & $4.9 \times 10^{-5}$ & $1.5 \times 10^{-5}$ & $3.2 \times 10^{-5}$ & 25 & $1.6 \times 10^{-5}$ & $3.3 \times 10^{-5}$ \\
\hline$A 2$ & 49 & 2.3 & 2.0 & 0.3 & 7.5 & 35 & 37 & $3.4 \times 10^{-5}$ & $3.1 \times 10^{-5}$ & $1.4 \times 10^{-5}$ & $3.4 \times 10^{-5}$ & $3.2 \times 10^{-5}$ & $1.4 \times 10^{-5}$ \\
\hline$A 3$ & 23 & 6.0 & 5.6 & 1.2 & & & & $2.1 \times 10^{-4}$ & $6.3 \times 10^{-5}$ & $4.4 \times 10^{-5}$ & $2.1 \times 10^{-4}$ & $6.3 \times 10^{-5}$ & $4.4 \times 10^{-5}$ \\
\hline BO & & & 22 & 4.7 & & & & 0.42 & 0.25 & 0.11 & 3.1 & 1.2 & 0.59 \\
\hline$B 1$ & & & $1.2 \times 10^{3}$ & 41 & & & & 3.6 & 0.73 & 0.29 & 10 & 4.2 & 2.6 \\
\hline$B 2$ & & & $3.4 \times 10^{4}$ & $2.2 \times 10^{2}$ & & & & 45 & 1.6 & 1.5 & 81 & 45 & 4.8 \\
\hline CO & & & 78 & 49 & & & & 1.0 & 0.5 & 0.2 & 6.1 & 1.3 & 0.87 \\
\hline$C 1$ & & & $2.1 \times 10^{3}$ & 93 & & & & 5.9 & 1.3 & 1.5 & 33 & 5.7 & 5.1 \\
\hline$C 2$ & & & $1.2 \times 10^{4}$ & $4.4 \times 10^{2}$ & & & & 23 & 4.5 & 2.1 & $1.8 \times 10^{2}$ & 29 & 13 \\
\hline DO & & & $1.7 \times 10^{2}$ & 28 & 11 & 21 & 50 & 2.8 & 0.89 & 0.51 & 21 & 5.1 & 2.9 \\
\hline$D 1$ & & & $4 \times 10^{2}$ & 19 & & & & 3.8 & 0.75 & 0.45 & 23 & 5.7 & 2.8 \\
\hline
\end{tabular}

Table 4 - flow_error as defined by equation (7) for the backbone of the fracture network types defined in Table 1 and for the three computational methods ANIS_GEO, TENSOR_SIM and EHM at different discretization levels. Domain discretization refers to the ratio of the domain size to the block size in each direction. EHM methods are characterized in brackets by their block-scale discretization parameter $r_{b l o c k}$ equal to the ratio expressed in $\%$ between the block-scale discretization distance $d_{\text {block }}$ and the block scale. Empty cells mean that the conditions of application of the method are not fulfilled in the corresponding case. 


\begin{tabular}{|c|c|c|c|c|c|c|c|c|c|c|c|c|c|c|}
\hline \multirow[b]{2}{*}{ Domain discretization } & \multirow[t]{2}{*}{ DFN } & \multicolumn{4}{|c|}{ ANIS_GEO } & \multicolumn{3}{|c|}{ TENSOR_SIM } & \multicolumn{3}{|c|}{ EHM (10\%) } & \multicolumn{3}{|c|}{ EHM $(25 \%)$} \\
\hline & & $10 \times 10$ & $30 \times 30$ & $50 \times 50$ & $200 \times 200$ & $10 \times 10$ & $30 \times 30$ & $50 \times 50$ & $10 \times 10$ & $30 \times 30$ & $50 \times 50$ & $10 \times 10$ & $30 \times 30$ & $50 \times 50$ \\
\hline$A 0$ & 26 & 0.46 & 4.4 & 12 & 200 & 1.2 & 12 & 34 & 14 & 110 & 140 & 1.2 & 42 & 75 \\
\hline$A 1$ & 26 & 0.46 & 4.4 & 12 & 200 & & & & 15 & 101 & 140 & 1.2 & 41 & 87 \\
\hline$A 2$ & 0.16 & 0.46 & 4.4 & 12 & 200 & 1.2 & 12 & 34 & 0.7 & 1.6 & 2.4 & 0.6 & 1.5 & 2.4 \\
\hline$A 3$ & 0.16 & 0.46 & 4.4 & 12 & 200 & & & & 0.7 & 1.6 & 2.4 & 0.6 & 1.5 & 2.4 \\
\hline BO & 0.87 & & & 12 & 200 & & & & 0.45 & 1.4 & 2.5 & 0.3 & 1.2 & 2.2 \\
\hline B1 & 13 & & & 12 & 200 & & & & 1.1 & 4.2 & 6.7 & 0.6 & 2.6 & 4.5 \\
\hline$B 2$ & 33 & & & 12 & 200 & & & & 1.5 & 6.6 & 12 & 0.7 & 3.5 & 7.1 \\
\hline CO & 6.1 & & & 12 & 200 & & & & 1.6 & 4.0 & 6.0 & 0.9 & 3.0 & 5.0 \\
\hline$C 1$ & 53 & & & 12 & 200 & & & & 4.5 & 16 & 26 & 1.8 & 8.6 & 16 \\
\hline$C 2$ & 240 & & & 12 & 200 & & & & 11 & 56 & 96 & 2.8 & 22 & 46 \\
\hline DO & 52 & & & 12 & 200 & 1.2 & 12 & 34 & 8.7 & 27 & 41 & 2.9 & 15 & 26 \\
\hline$D 1$ & 51 & & & 12 & 200 & & & & 8.7 & 27 & 41 & 2.9 & 15 & 26 \\
\hline
\end{tabular}

692 Table 5 - Numerical memory complexity expressed as the number of non-zero elements (nnz) of the domain-scale linear systems issued from the

693 flow discretization for the network cases of Table 1. Parameters are identical to those of Table 4. All numbers are expressed in thousands of nonzero elements. 


\begin{tabular}{|c|c|c|c|c|c|c|}
\hline & \multicolumn{3}{|c|}{ EHM (10\%) } & \multicolumn{3}{c|}{ EHM (25\%) } \\
\hline Domain discretization & $\mathbf{1 0 \times 1 0}$ & $\mathbf{3 0 \times 3 0}$ & $\mathbf{5 0 \times 5 0}$ & $\mathbf{1 0 \times \mathbf { 1 0 }}$ & $\mathbf{3 0 \times 3 0}$ & $\mathbf{5 0 \times 5 0}$ \\
\hline $\boldsymbol{A 0}$ & 70 & 21 & 14 & 91 & 53 & 36 \\
\hline $\boldsymbol{A 1}$ & 69 & 23 & 14 & 91 & 53 & 32 \\
\hline $\boldsymbol{A 2}$ & 4.7 & 1.7 & 2.6 & 13 & 4.3 & 2.6 \\
\hline $\boldsymbol{A 3}$ & 4.7 & 1.7 & 2.6 & 13 & 4.3 & 2.6 \\
\hline $\boldsymbol{B 0}$ & 14 & 7 & 5.3 & 26 & 14 & 10 \\
\hline $\boldsymbol{B 1}$ & 44 & 23 & 15 & 59 & 37 & 28 \\
\hline $\boldsymbol{B 2}$ & 54 & 32 & 23 & 70 & 49 & 39 \\
\hline $\boldsymbol{C 0}$ & 25 & 12 & 8.3 & 41 & 22 & 15 \\
\hline $\boldsymbol{C 1}$ & 53 & 29 & 21 & 70 & 46 & 35 \\
\hline $\boldsymbol{C 2}$ & 72 & 44 & 33 & 86 & 65 & 53 \\
\hline $\boldsymbol{D} \boldsymbol{D}$ & 50 & 26 & 18 & 70 & 44 & 32 \\
\hline $\boldsymbol{D 1}$ & 50 & 26 & 18 & 70 & 44 & 32 \\
\hline
\end{tabular}

696

697 Table 6 - Block-scale border merging percentage $p_{\text {border }}$ for the fracture network types of Table 1. 


\begin{tabular}{|c|c|c|c|c|c|c|c|c|c|c|c|c|c|c|}
\hline & \multicolumn{4}{|c|}{ ANIS_GEO } & \multicolumn{3}{|c|}{ TENSOR_SIM } & \multicolumn{3}{|c|}{ EHM (10\%) } & \multicolumn{3}{|c|}{ EHM (25\%) } & \multirow[t]{2}{*}{ Table 2} \\
\hline Domain discretization & $10 \times 10$ & $30 \times 30$ & $50 \times 50$ & $200 \times 200$ & $10 \times 10$ & $30 \times 30$ & $50 \times 50$ & $10 \times 10$ & $30 \times 30$ & $50 \times 50$ & $10 \times 10$ & $30 \times 30$ & $50 \times 50$ & \\
\hline DO & & & $5.5 \times 10^{2}$ & $1.5 \times 10^{2}$ & 37 & 38 & 14 & 11 & 4.9 & 3.0 & 76 & 23 & 14 & \\
\hline$D 1$ & & & $1.7 \times 10^{3}$ & $1.5 \times 10^{2}$ & & & & 12 & 4.3 & 2.4 & 70 & 20 & 11 & I4' \\
\hline
\end{tabular}

698

699 Table 7 - flow_error as defined by equation (7) for the infinite cluster of fracture networks $D 0$ and $D 1$ (see Table 1 for description). EHM

700 methods are characterized in brackets by their block-scale discretization parameter $r_{\text {block }}$ equal to the ratio expressed in $\%$ of the block-scale

701 discretization distance $d_{\text {block }}$ to the block scale. 


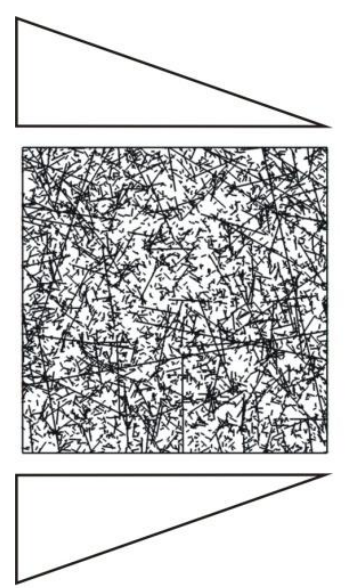

(a)

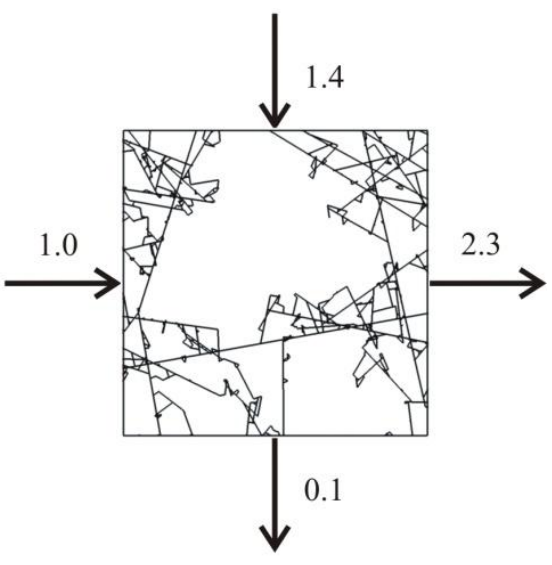

(b)

704 Figure 1 - Fracture network at the block scale (a) and corresponding flows (b) for the gradient head 705 boundary conditions illustrated in (a). Fracture network parameters are the system size $L$ normalized 706 by the smallest fracture length $(L=100)$, the fracture density number twice larger as its value at 707 percolation threshold, the power-law fracture length exponent of 2.5 and the lognormal 708 transmissivity distribution of logarithmic standard deviation 3.0. Boundary flows integrated on the 709 domain sides and normalized by the mean fracture transmissivity are given in (b). They display large 710 differences between opposite sides and illustrate the non-tensor nature of the flows. 


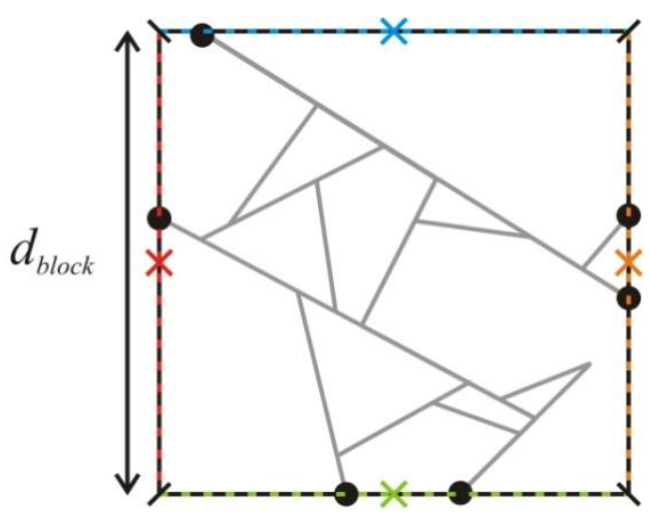

(a)

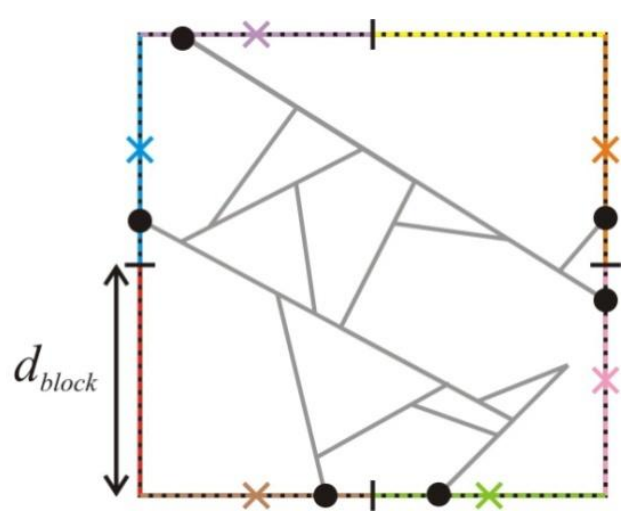

(b)

712 Figure 2 - Principle of the block-border discretization with two different discretization scales $d_{\text {block }}$

713 corresponding to the side length (a) and to half of it (b). The backbone of the sub-network contained

714 in the block is represented by the grey segments. Intersections $\boldsymbol{m}_{\mathrm{k}}$ between the backbone and the

715 block borders are the black dots. Discretization segments and poles $\boldsymbol{p}_{\boldsymbol{k}}$ are respectively the color

716 dashed segments and crosses. In (a), the four discretization segments intersect the backbone in one or

717 two points. The four poles corresponding to the four crosses are thus defined and the Equivalent

718 Hydraulic Matrix (EHM) is of rank 4. In (b), only six of the eight discretization segments intersect

719 the backbone leading to the definition of 6 poles and to an EHM of rank 6. 


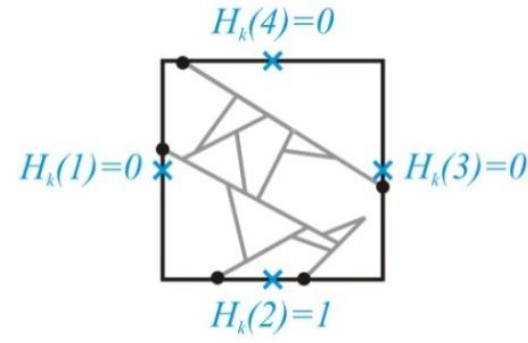

(a)

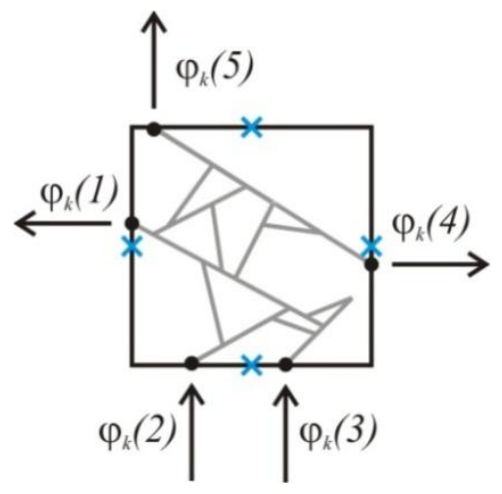

(c)

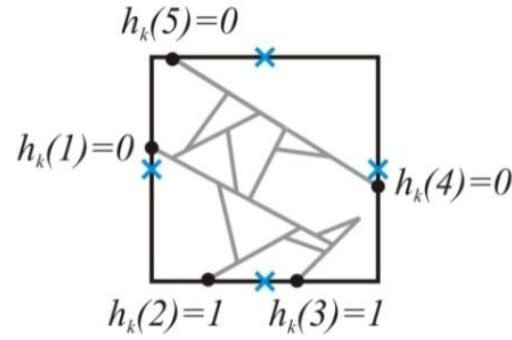

(b)

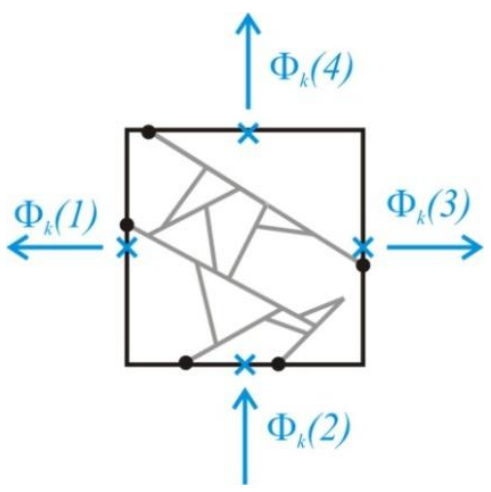

(d)

$$
\left\{\begin{array}{l}
A_{k}(2,1)=\phi_{k}(1) \\
A_{k}(2,2)=\phi_{k}(2) \\
A_{k}(2,3)=\phi_{k}(3) \\
A_{k}(2,4)=\phi_{k}(4)
\end{array}\right.
$$

(e)

720 Figure 3 - Principle of the determination of one of the columns of the Equivalent Hydraulic Matrix

$721 \boldsymbol{A}_{\boldsymbol{k}}$. In this example, block $k$ is made up of fives intersections between the sub-network and the block 722 borders (black points) and four poles (blue crosses). The boundary conditions applied to poles 723 illustrated in (a) are a fixed head of 1 for the $2^{\text {nd }}$ pole and 0 for the other ones. They condition the 724 boundary conditions applied to the intersections illustrated in (b), which are a fixed head of 1 for the 725 intersections represented by the $2^{\text {nd }}$ pole and 0 for the other ones. Flow rates in poles (d) are deduced 726 from flow rates at the intersections (c). The flow rate at the $i^{\text {th }}$ pole is the sum of the flow rates at the 727 intersections represented by this pole. The elements of the second column of the matrix $\boldsymbol{A}_{\boldsymbol{k}}$ are 728 deduced from flow rates computed at the poles (e). 


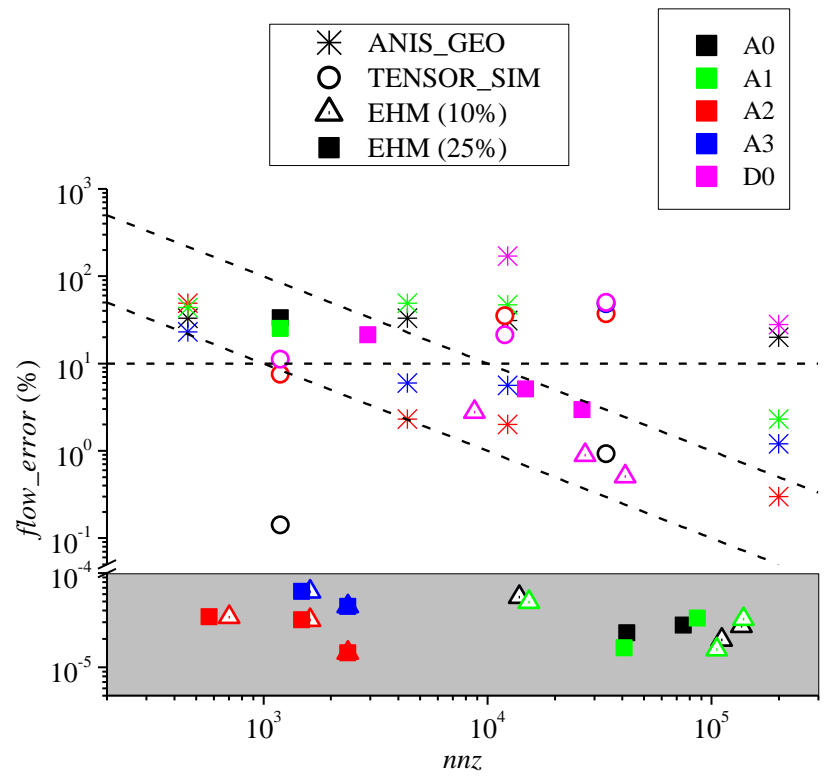

731 Figure 4 - flow_error versus numerical memory complexity ( $n n z)$ for lattice structures and dense

732 fracture networks with constant fracture transmissivity (magenta symbols). The grey area underlines

733 a lower part of the graph where errors range between $5 \times 10^{-6} \%$ and $10^{-4} \%$. The dashed horizontal line

734 pictures the $10 \%$ error value. The dashed diagonal lines are power-law functions of exponent -1 and 735 are meant as a guide for the eye for the decrease tendency of the EHM method. Note that errors 736 larger than $10^{3}$ are not represented. 

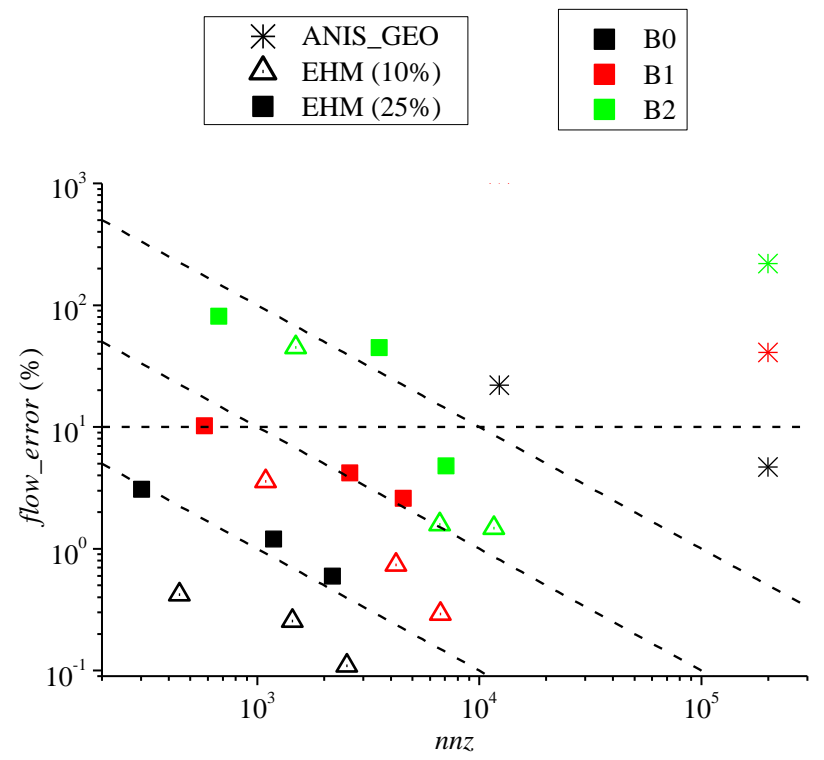

739 Figure 5 - flow_error versus numerical memory complexity (nnz) for stochastic complex fracture 740 networks at threshold with distributed fracture transmissivities. The dashed horizontal line pictures 741 the $10 \%$ error value. The dashed diagonal lines are power-law functions of exponent -1 and are 742 meant as a guide for the eye for the decrease tendency of the EHM method. Note that errors larger 743 than $10^{3}$ are not represented. 


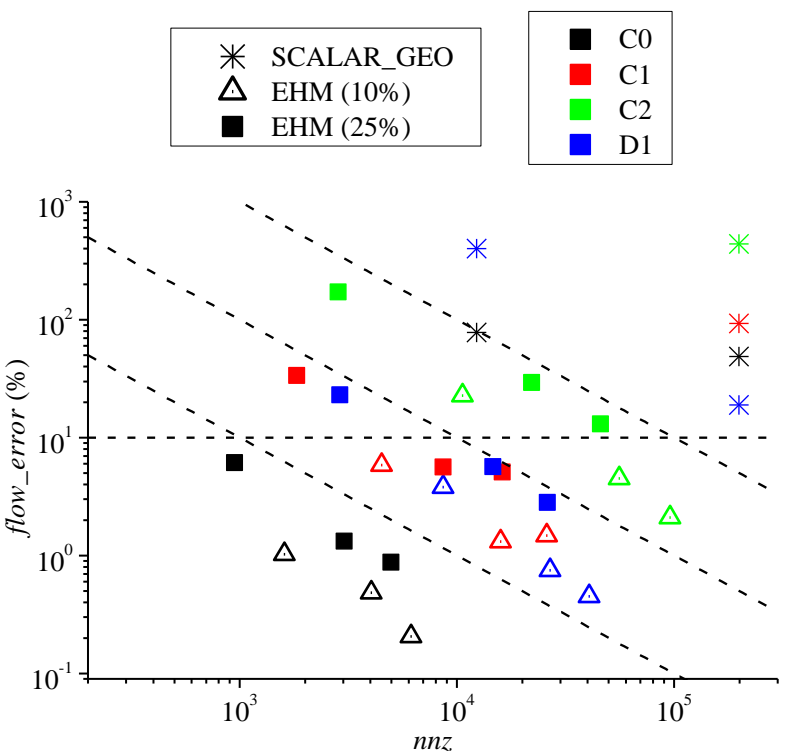

745

746 Figure 6 - flow_error versus numerical memory complexity (nnz) for stochastic complex fracture

747 networks with distributed fracture transmissivities. The dashed horizontal line pictures the $10 \%$ error 748 value. The dashed diagonal lines are power-law functions of exponent -1 and are meant as a guide

749 for the eye for the decrease tendency of the EHM method. Note that errors larger than $10^{3}$ are not 750 represented. 


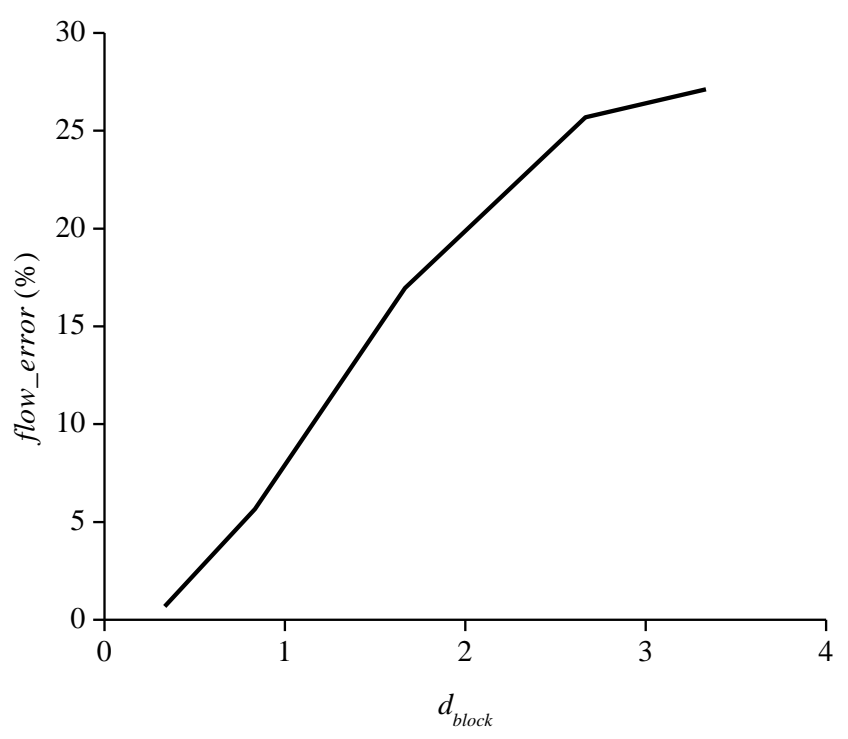

753

754 Figure 7 -flow_error versus $d_{\text {block }}$ the discretization ratio $r_{\text {block }}$ times the length of the block edge for 755 the network $D 1$ (domain size $L=100$ ). 


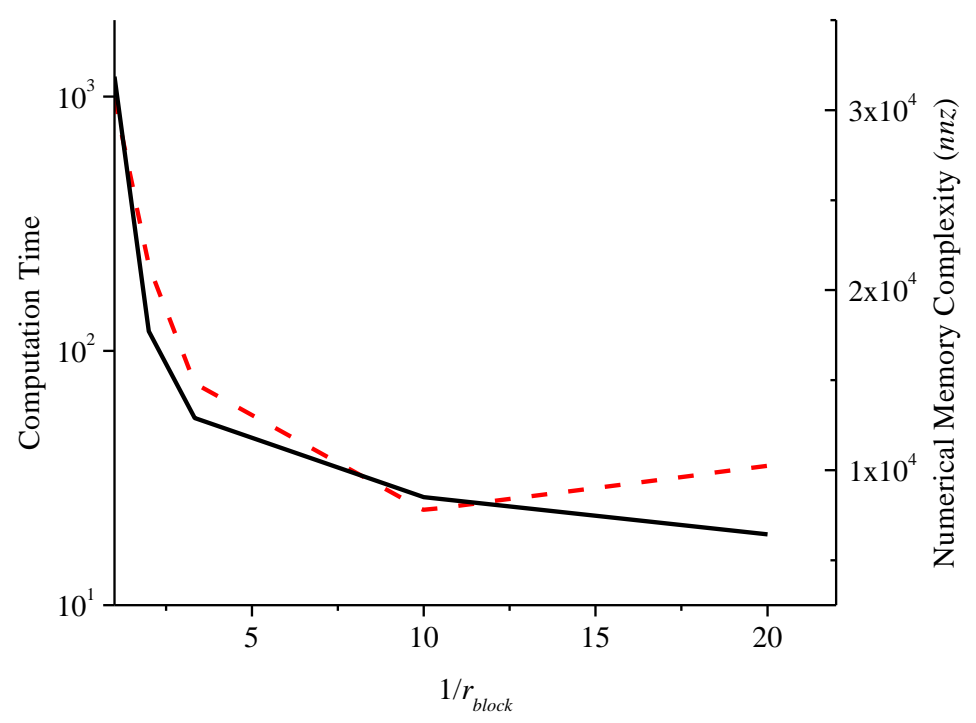

759 Figure 8 - Computation time (red dashed line) and numerical memory complexity taken as the 760 number of non-zero elements in the largest matrix (black line) as a function of block size divided by 761 the segment discretization length $1 / r_{\text {block }}$ for $D 1$ with $d_{\text {block }}$ equal to 1 . 